Article

\title{
Electron-Impact Widths and Shifts of B III 2p-2s Lines
}

\author{
Bin Duan ${ }^{1, *}$, Muhammad Abbas Bari ${ }^{2}$, Zeqing Wu ${ }^{1}$ and Jun Yan ${ }^{1}$ \\ ${ }^{1}$ Institute of Applied Physics and Computation Mathematics, Beijing 100088, China; \\ E-Mails:wu_zeqing@iapcm.ac.cn; yan_jun@iapcm.ac.cn \\ ${ }^{2}$ Pakistan Atomic Energy Commission, P. O. Box 1114, Islamabad 44000, Pakistan; \\ E-Mail: kmabari@gmail.com
}

* Author to whom correspondence should be addressed; E-Mail: alexduan1967@ hotmail.com;

Tel.: +86-10-6193-5108; Fax: +86-10-6201-0108.

Received: 17 March 2014; in revised form: 23 April 2014 / Accepted: 5 May 2014 /

Published: 15 May 2014

\begin{abstract}
In this paper, we present results for the relativistic quantum mechanical calculations of electron-impact line widths and shifts of $2 \mathrm{p}-2 \mathrm{~s}$ transitions in doubly ionized boron (B III) ions. We use the Dirac R-matrix methods to solve $(N+1)$-electron colliding systems for the scattering matrices that are required. The line widths are calculated for an electron density $1.81 \times 10^{18} \mathrm{~cm}^{-3}$ and electron temperature $10.6 \mathrm{eV}$. The obtained results agree well with all the semiempirical calculations and most of the semiclassical calculations, and are closer to the experimental results published by Glenzer and Kunze (Glenzer, S.; Kunze, H.-J. Stark broadening of resonance transitions in B III. Phys. Rev. A 1996, 53, 2225-2229). Our line widths are almost twice as large as the earlier quantum mechanical calculations for the set of particular plasma conditions.
\end{abstract}

Keywords: electron-impact widths and shifts; $(N+1)$-electron colliding systems; line shapes

\section{Introduction}

The broadening of spectral lines due to collisions with charged particles appears to be important in the studies on the behavior of atomic interactions, and is indispensable for interpreting the spectra of astrophysical and laboratory plasmas. In atmospheres of moderately hot stars (A-type star) to very hot (B- and O-type) stars and in white dwarfs, a large number of isolated ion lines has been observed [1] 
and Stark broadening is the well proven dominant pressure-broadening mechanism especially for white dwarfs [2]. The knowledge of Stark broadening parameters is crucial for astrophysical applications. The electron-impact broadening data of width and shift for a large number of lines of various elements and their ions are required for radiation transport in stellar plasma. Dimitrijević and Sahal-Bréchot [3] pointed out that line profiles of Boron in various ionization stages play an important role for accurate radiative opacity calculations [4,5]. Moreover, Stark broadening parameters of B III lines are of prime interest not only for the investigation and diagnosis of laboratory and laser-produced plasma, but also for the research of regularities and systematic trends. Consequently, numerous experimental and theoretical studies of Stark widths and shifts of spectral lines have been undertaken since the fundamental and pioneering works by Baranger [6-8]. In fact, Stark broadening parameters have been extensively calculated with both the sophisticated semiclassical [9] and quantum-mechanical methods, although they often require a considerable effort even for the evaluation of a single line width and shift. Apart from the several successful semiclassical frameworks [9-13] and semi-empirical formula [13-15] for performing calculations of electron-impact broadening parameters, it is equally important to perform detailed and systematic quantum mechanical calculations to provide a quantitative check of the different approximations involved in the usual semi-classical methods.

As a continuation of our previous papers [16,17], the aim of present paper is two-fold. First, we want to extend the results of our previous quantum calculations in the impact approximation to investigate the validity and accuracy of our quantum mechanical approach for more spectral lines of the large number of elements. Second, accurate line profile measurements of the resonance doublet transitions $2 p^{2} P_{3 / 2,1 / 2}^{o} \rightarrow 2 s^{2} S_{1 / 2}$ in Li-like boron were performed by Glenzer and Kunze [18]. These experimental results of Stark widths of the investigated transitions are found to disagree substantially with various theoretical calculations $[3,11,12,19,20]$ even for the advanced quantum-mechanical calculations $[4,5,21,22]$. For instance, the experimental Stark width of the B III $2 s-2 p$ resonance doublet was found to be about a factor of two larger than the convergent close coupling (CCC) and Coulomb-Born exchange (CBE) calculations [21]. This disagreement makes it necessary that systematic theoretical calculations of line shifts and widths in B III ions in the particular selected plasma conditions should be carried out using our new quantum mechanical approach. Furthermore, the agreement obtained between our quantum results $[16,17]$ within the framework of impact approximation theory and the available experimental data encourages us to perform calculations for the $2 p^{2} P_{3 / 2,1 / 2}^{o} \rightarrow 2 s^{2} S_{1 / 2}$ resonance transitions in B III ions. In the present work, we use the fully relativistic Dirac R-matrix codes [23] (DARC from hereafter) to calculate electron-impact collision strengths $\Omega$ and scattering matrices of B III ions. The target states of B III ions are determined for the low lying levels up to $n=5$ by employing the General Purpose Relativistic Atomic Structure Package (GRASP2) [24,25] (partly improved by us). The rest of this paper is organized as follows. An outline of the basic procedure for our calculations is presented in section II. In Section III, our results are compared and discussed with available experimental and theoretical data. The conclusions are summarized in section IV. 


\section{Computational Procedure}

We briefly outline here the essential features of the computational procedure where the values of some relevant parameters are presented for the sake of completeness. More details of our computational formalism to address electron-impact broadening parameters have been outlined elsewhere [16,17,26,27], and will not be detailed here. By using DARC, a fully relativistic package, the width and shift of spectral lines due to electron collisions are written as [4,5,28-30]

$$
w+i d=\alpha N_{e} \int_{0}^{\infty} T_{e}^{-3 / 2} \exp (-\varepsilon / T) \Omega(\varepsilon) d \varepsilon .
$$

where $\alpha=2.8674 \times 10^{-23} \mathrm{eV} \mathrm{cm}^{3}, T_{e}$ is the electron temperature in $\mathrm{eV}, N_{e}$ is the electron density in $\mathrm{cm}^{-3}$ and $\varepsilon$ is the energy of colliding electron in Rydberg. In above expression, $w$ and $d$ represent the half-width( a half of full width at half-maximum) and shift of spectral line in eV, respectively. The dimensionless collision strength $\Omega(\varepsilon)$ as a function of energy of colliding electrons is evaluated in $\mathrm{j}-\mathrm{j}$ coupling scheme according to Equation (2) of [17]. The computational details about the collision strength of $(N+1)$-electron colliding systems (colliding electron+target ion) can be found in [17]. The shift $d$ in Equations (1) and (2) of [16] describes that a negative shift $d$ in photon energy always corresponds to red shift in wavelength, which corresponds to a small reduction in the energy of the transition line.

In order to calculate width and shift of spectral lines $2 p \rightarrow 2 s$ in B III ions, we calculate atomic structure data from 13 non-relativistic configurations $1 s^{2} n l(n \leq 5, l \leq n-1)$ which give rise to 22 bound states. The relative data of B III 2p-2s lines are shown in Table 1 . The lowest 8 bound states are selected as the target states. The relativistic orbitals generated by the GRASP 2 code are then used by the DARC package to construct the colliding system that consists of upper or lower state of the desired transition in target ion and a colliding free-electron. In our calculations, the colliding electron is limited by two constraints. First, the quantum number of the orbital angular momentum is $\ell \leq 25$, and second, the total number of its continuum basis function for a given $\kappa(\kappa=-\ell-1$, if $\kappa<0$, else $\kappa=\ell$ ) is 30 . The required $\mathbf{K}$ - matrices and their corresponding symmetry information of the colliding system is obtained by using DARC. Since the dimensionless collision strength $\Omega(\varepsilon)$ defined in Equation (2) [17] is a function of incident electron-impact energies $\varepsilon$ and is computed in an increasing energy sequence with an energy increment $\Delta \varepsilon=0.008$ Rydberg by repeating the same procedure. Finally, we obtain the width and shift of spectral lines by solving Equation (1) numerically by means of trapezoidal integration rule.

Table 1. The relative atomic structure data of B III 2p-2s lines are obtained by General Purpose Relativistic Atomic Structure Package (GRASP2) [24,25].

\begin{tabular}{|c|c|c|c|c|}
\hline \multirow[t]{2}{*}{ Lines } & \multirow{2}{*}{$\begin{array}{c}\text { Energy of Lower State } \\
(\mathrm{eV})\end{array}$} & \multirow{2}{*}{$\begin{array}{c}\text { Energy of Upper State } \\
(\mathrm{eV})\end{array}$} & \multicolumn{2}{|c|}{ Oscillator Strength $g_{i} f_{i f}$} \\
\hline & & & Coulomb Gauge & Babushkin Gauge \\
\hline $2 p^{-} \rightarrow 2 s$ & -636.1998 & -630.1390 & 0.2768 & 0.2503 \\
\hline $2 p^{+} \rightarrow 2 s$ & -636.1998 & -630.1351 & 0.5543 & 0.5010 \\
\hline
\end{tabular}




\section{Numerical Results and Discussion}

The calculated electron-impact broadened full width at half-maximum $(\mathrm{FWHM}=2 w)$ and shifts in the B III ( $2 p \rightarrow 2 s)$ doublet transition lines as a function of electron temperature for an electron density of $1.81 \times 10^{18} \mathrm{~cm}^{-3}$ are displayed in Figure 1. Figure 1 clearly demonstrates that widths and shifts of doublet transition lines $2 p^{2} P_{3 / 2,1 / 2} \rightarrow 2 s^{2} S_{1 / 2}$ have very similar behavior with the increase of temperature. One can see that the line broadening parameters decrease with an increase in temperature. We find that electron-impact broadened full-widths $(2 w)$ are always much larger than the shifts of both the spectral lines for any given plasma condition. An example is provided at an electron temperature $T_{e}=10.6 \mathrm{eV}$, the calculated FWHM $(2 w)$ and shift $d$ for the transition line $2 p^{2} P_{3 / 2} \rightarrow 2 s^{2} S_{1 / 2}$ are 0.212 and 0.0517 (about 4 times higher), respectively, whereas for the transition line $2 p^{2} P_{1 / 2} \rightarrow 2 s^{2} S_{1 / 2}, 2 w=0.216$ and $d=0.0518$.

Figure 1. The electron-impact widths (FWHM) and shifts of doublet transitions $(2 p \rightarrow 2 s)$ in B III ions as a function of electron temperature for an electron density of $1.81 \times 10^{18} \mathrm{~cm}^{-3}$. (a) $2 p^{2} P_{3 / 2} \rightarrow 2 s{ }^{2} S_{1 / 2}$ (b) $2 p^{2} P_{1 / 2} \rightarrow 2 s{ }^{2} S_{1 / 2}$.
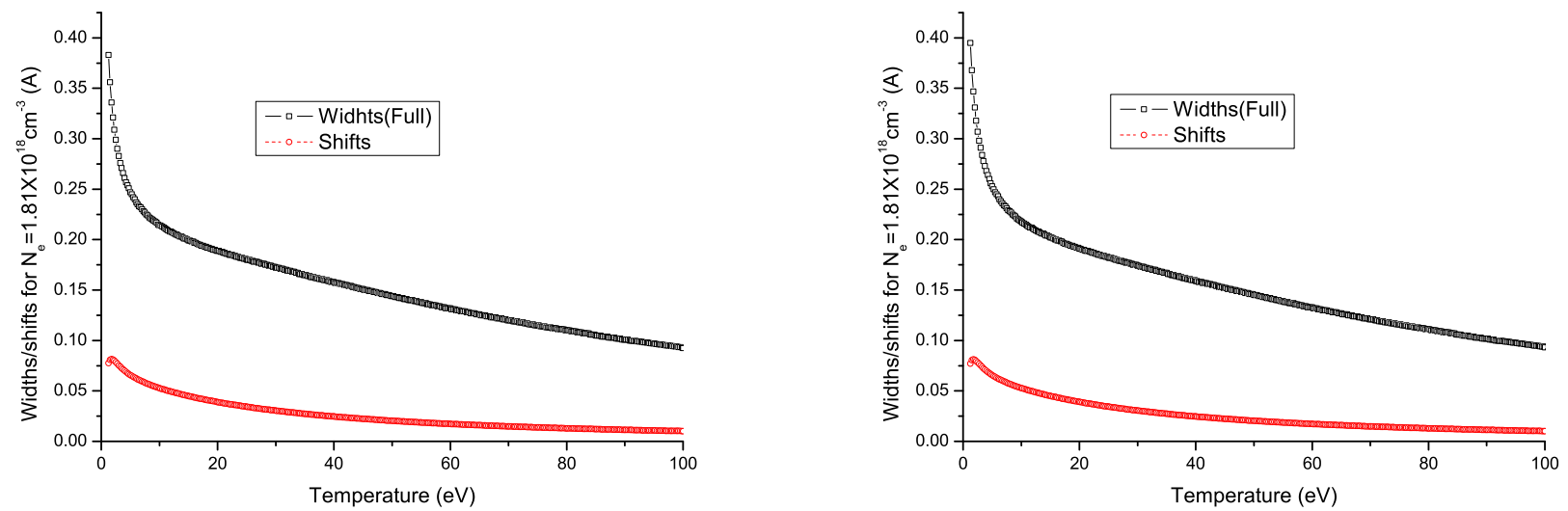

As previously mentioned, experimental Stark linewidths were measured by Glenzer and Kunze [18] by performing an accurate line profile measurements of the $2 p \rightarrow 2 s$ fine-structure components of the resonance doublet in Li-like boron. The authors used a homogeneous plasma region in a gas-liner pinch discharge, and plasma parameters (local electron density and ion temperature) were determined independently by $90^{\circ}$ collective Thomson scattering. The Stark linewidths (FWHM) of doublet transitions in B III were measured to be $2 w=0.22$ for an electron density $N_{e}=1.81 \times 10^{18} \mathrm{~cm}^{-3}$ and electron temperature $T_{e}=10.6 \mathrm{eV}$. Comparison reveals that measured linewidths agree very well with our presented values (within $3.6 \%$ accuracy).

In Table 2 our calculated values are compared both with the experimental data [18] and with various theoretical calculations [3-5,9,11,12,19-21] for an electron temperature of $T_{e}=10.6 \mathrm{eV}$ and an electron density of $N_{e}=1.81 \times 10^{18} \mathrm{~cm}^{-3}$. Table 2 contains theoretical widths $w_{t h}$ calculated on the basis of various theoretical approaches discussed ahead. In this table, the last column displays our quantum mechanical values of line widths for the doublet transitions in B III. It should be noted that both the semiclassical calculations $[3,20]$ agree well with the experimental measurements. 
Historically, Griem [14] provided a simplified semi-empirical method with the use of an effective Gaunt-factor to calculate halfwidths of an isolated ion line broadened by electron-impact. The ratios of measured Stark widths [18] for the 2p-2s transitions of B III to the semi-empirically [9] calculated ones are 1.19 (see Table 2) and are noticeably different from the semi-classically calculated ones. Later in 1980, Dimitrijević and Konjević modified this well known semi-empirical approach by considering dipole-allowed electron collisions and by selecting the Gaunt factor as a suitable empirical value [15]. Hey and Breger [19] performed calculations based on the quasi-classical Gaunt factor approximation different from Griem [9] to calculate the minimum and maximum impact parameters of the electron collision process.

Table 2. Ratio of experimental electron-impact half-widths $w_{\text {exp }}$ [18] of $2 \mathrm{p}-2 \mathrm{~s}$ line in B III to different theoretical half-widths $w_{t h}$ for an electron temperature of $T_{e}=10.6 \mathrm{eV}$ and an electron density of $N_{e}=1.81 \times 10^{18} \mathrm{~cm}^{-3}$. The letters from a to h denote theoretical widths $w_{t h}$ calculated on the basis of various theoretical approaches; a: semiempirical calculations [9]; b: Semiempirical calculations [19]; c: semiclassical calculations [3]; d: semiclassical calculations [20]; e: semiclassical calculations (NPSCII) [11,12]; f: R-matrix calculations [4,5]; $\mathbf{g}$ and $\mathbf{h}$ : calculations of $\mathrm{CCC}$ and $\mathrm{CBE}$ methods, respectively [21].

\begin{tabular}{ccccccccccc}
\hline$w_{\text {exp }} / w_{t h}$ & Wavelength $(\mathbf{0 . 1} \mathbf{~ n m})$ & $\mathbf{a}$ & $\mathbf{b}$ & $\mathbf{c}$ & $\mathbf{d}$ & $\mathbf{e}$ & $\mathbf{f}$ & $\mathbf{g}$ & $\mathbf{h}$ & Present \\
\hline $2 p^{2} P_{3 / 2} \rightarrow 2 s^{2} S_{1 / 2}$ & 2065.78 & 1.19 & 1.24 & 1.03 & 1.09 & 1.77 & 1.81 & 2.1 & 1.9 & 1.04 \\
$2 p^{2} P_{1 / 2} \rightarrow 2 s^{2} S_{1 / 2}$ & 2067.24 & 1.19 & 1.24 & 1.02 & 1.08 & 1.76 & 1.79 & 2.1 & 1.9 & 1.02 \\
\hline
\end{tabular}

By using the semiclassical perturbation formalism [31], Dimitrijević and Sahal-Bréchot studied and also devoted a series of papers to the calculations of Stark broadening parameters of spectral lines of multicharged ions more than a dozen times. In particular, they studied the line widths and shifts for Be II and B III spectral lines due to interactions with electrons, protons and singly ionized helium ions [3]. The nonperturbative semiclassical methods (NPSC) were introduced and formulated by Alexiou [11,12], which are characterized by choosing and defining a minimum impact parameter $\rho_{\min }(v)$ of collisions. These NPSC results show excellent agreement with the highest quality experimental data from the Bochum gas-liner pinch [32,33] for a number of measured isolated lines as exhibited by the results of Dimitrijević and Sahal-Bréchot [3]. Alexiou and Lee [11,12] further improved the NPSC by dropping the long-range approximation and using the full Coulomb interaction, and named it NPSCII. In the case of B III $2 p \rightarrow 2 s$ lines, NPSCII yielded the values of FWHM which are $10 \%$ larger than that of the R-matrix calculations performed by Seaton [4,5] and 5\% smaller than that of semiempirical results [21]. The NPSCII results are in excellent agreement with these two quantal calculations.

In contrast to the case of semiclassical calculations, almost all of the quantum-mechanical calculations of doublet transitions ( $2 p \rightarrow 2 s$ ) of Li-like B are about half of the corresponding experimental data [18], even though they are consistent with each other. The widths obtained with five-state close-coupling quantum mechanical calculations [4,5] are about half of the experimental values (differ by a factor $\approx 2$ ). In addition to the $\mathrm{CC}$ work, Griem et al. [21] performed calculations for Stark broadening parameters of the B III $2 p-2 s$ lines with the convergent close coupling (CCC) and the Coulomb-Born exchange 
(CBE) methods. These two sets of linewidths are in good agreement with each other as well as with previous quantum mechanical R-matrix calculations [4,5].

Finally, the ratio of measured-to-calculated widths tabulated by us (Table 2) for the various theoretical calculations provide guidance on the degree of agreement between the experimental data and theory, and thus provide a valuable indication of the quality of the calculated as well as measured data. It is seen that our calculations along with semiclassical calculations compare well with the experimental data [18], which was also observed in earlier articles. Our calculated widths are about twice as large as the earlier mentioned quantum mechanical calculations, including the NPSCII formalism as well.

\section{Conclusions}

We have performed fully quantum mechanical calculations of electron-impact widths and shifts of the B III $(2 p \rightarrow 2 s)$ doublet transition lines by employing GRASP2 and DARC atomic packages for plasmas under electron temperature in the range $(0 \sim 100 \mathrm{eV})$. We have found that our quantum results agree well with both the semiempirical and semiclassical theory, and are more closer to the experimental ones. Our calculations are generally more consistent with the semiclassical theory and are about twice as large as other quantum mechanical ones. This is not similar to what had already been predicted with quantum mechanical calculations for Stark broadening parameters for B III ions [21]. Unfortunately, we still can not answer these puzzling discrepancies existing among these theoretical calculations. Therefore, comparison of the theory with experiment indicate that further studies and improvements of the theory for more new transition lines of boron ions are required. However, just for the values, our results are much closer to the measured values as compared to the other theoretical calculations. Finally, we believe that our results are useful for new plasma diagnostics.

\section{Acknowledgements}

This work is supported by the National Natural Science Foundation of China (NSFC) (Grant No. 11275029 and No. 11204017)) and Foundation for Development of Science and Technology of Chinese Academy of Engineering Physics (Grant No. Grant No. 2013A0102005). One of us is gratefully indebted to the support of IAEA too.

\section{Author Contributions}

B. D. contributed to the original theory calculation and analysis. The other authors performed supporting calculation and the data analysis. All authors were involved in the discussion of results and commented on the manuscript.

\section{Conflicts of Interest}

The authors declare no conflict of interest. 


\section{References}

1. Dimitrijević, M.S. Stark broadening in astrophysics (Applications of Belgrade school results and collaboration with Soviet republics). Astron. Astrophys. Trans. 2003, 22, 389-412.

2. Sahal-Bréchot, S. Case studies on recent Stark broadening calculations and STARK-B database development in the framework of the European project VAMDC (Virtual Atomic and Molecular Data Center). J. Phys. Conf. Ser. 2010, 257, 012028.

3. Dimitrijević, M.S.; Sahal-Bréchot, S. Stark broadening of specral lines of multicharged ions of astrophysical interest. XIV Be III and B III. Astrophys. Suppl. Ser. 1996, 119, 369-371.

4. Seaton, M.J. Atomic data for opacity calculations: V. Electron impact broadening of some CIII lines. J. Phys. B 1987, 20, 6431-6446.

5. Seaton, M. J., Atomic data for opacity calculations: VIII. Line-profile parameters for 42 transitions in Li-like and Be-like ions. J. Phys. B 1988, 21, 3033-3053.

6. Baranger, M. Simplified quantum-mechanical theory of pressure broadening. Phys. Rev. 1958, 111, 481-492.

7. Baranger, M. Problem of overlapping lines in theory of pressure broadening. Phys. Rev. 1958, 111, 494-504.

8. Baranger, M. General impact theory of pressure broadening. Phys. Rev. 1958, 112, 855-865.

9. Griem, H.R. Spectral Line Broadening by Plasmas; Academic Press: New York, NY, USA, 1974.

10. Sahal-Bréchot, S. Impact theory of the broadening and shift of specral lines due to electrons and ions in plasma. Astron. Astrophys. 1969, 1, 91-123.

11. Alexiou, S.; Lee, R.W. Electron line broadening in plasmas: Resolution of the quantum vs. semiclassical calculations puzzle. In Proceedings of the AIP Conference Proceedings, Berlin, Germany, 10-14 July 2000; Volume 559, pp. 35-143; doi:10.1063/1.1370605.

12. Alexiou, S. Review of Isolated Lines. In Proceedings of the 2nd Spectral Line Shapes in Plasmas Code Comparison Workshop, Vienna, Austria, 5-9 August, 2013.

13. Griem, H.R. Plasma Spectroscopy; McGraw-Hill: New York, NY, USA, 1964; pp. 129; 445.

14. Griem, H.R. Semiempirical Formulas for the Electron-Impact Widths and Shifts of Isolated Ion Lines in Plasmas. Phys. Rev. 1968,165, 258-266.

15. Dimitrijević, M.S.; Konjević, N. Starkwidths of doubly and triply ionized atom lines. J. Quant. Spectrosc. Radiat. Transf. 1980, 24, 451-459.

16. Duan, B.; Bari, M.A.; Wu, Z.Q.; Yan, J.; Li, Y.M.; Wang, J.G. Relativistic quantum mechanical calculations of electron-impact broadening for spectral lines in Be-like ions. Astron. Astrophys. 2012, 547, A4.

17. Duan, B.; Bari, M.A.; Wu, Z.Q.; Yan, J.; Li, Y.M. Widths and shifts of spectral lines in He II ions produced by electron impact. Phys. Rev. A 2012, 86, 052502:1-052502:6

18. Glenzer, S.; Kunze, H.-J. Stark broadening of resonance transitions in B III. Phys. Rev. A 1996, $53,2225-2229$.

19. Hey, J.D.; Breger, P. Calculated stark widths of oxygen ion lines. J. Quant. Spectrosc. Radiat. Transf. 1980, 24, 349-364. 
20. Alexiou, S. In Proceedings of the 13th International Conference on Spectral Line Shapes, Firenze, Italy, 16-21 June 1996; Zoppi, M., Ulivi, L., Eds.; AIP Conf. Proc. No. 386; AIP: New York, NY, USA, 1997; pp. 79-98.

21. Griem, H.R.; Ralchenko, Y.V.; Bray, I. Stark broadening of the B III 2s-2p lines. Phys. Rev. E 1997, 56, 7186-7192.

22. Stambulchik, E.; Maron, Y. A study of ion-dynamics and correlation effects for spectral line broadening in plasma: K-shell lines. J. Quant. Spectrosc. Radiat. Transf. 2006, 99, 730-749.

23. Norrington, P. DARC manual. Available online: http://www.am.qub.ac.uk /users/p.norrington (DARC-20040123.tar) (accessed on 23 April 2014).

24. Dyall, K.G.; Grant, I.P.; Johnson, C.T.; Parpia, F.A. ; Plummer, E.P. GRASP: A general-purpose relativistic atomic structure program. Comput. Phys. Commun. 1989, 55, 425-456.

25. Duan, B.; Bari, M.A.; Zhong, J.Y.; Yan, J.; Li, Y.M.; Zhang, J. Energy levels and radiative rates for optically allowed and forbidden transitions of Ni XXV ion. Astron. Astrophys. 2008, 488, 1155-1157.

26. Duan, B.; Bari, M.A.; Wu, Z.Q.; Yan, J.; Li, Y.M. Electron-impact widths and shifts of Sr II lines in ultracold neutral plasmas. Phys. Rev. A 2013, 87, 032505:1-032505:5.

27. Duan, B.; Bari, M.A.; Wu, Z.Q.; Yan, J.; Li, Y.M. Electron-impact broadening parameters for Be II, Sr II, and Ba II spectral lines. Astron. Astrophys. 2013, 555, A144.

28. Bely, O.; Griem, H.R. Quantum-mechanical calculations for the electron-impact braodening of resonance lines of singly ionized magnesium. Phys. Rev. A 1970, 1, 97-103.

29. Peach, G. Theory of the pressure broadening and shift of spectral lines. Adv. Phys. A 1981, 30, 367-474.

30. Elabidi, H.; Nessib, N.B.; Cornille, M.; Dubau J.; Sahal-Brechot, S. Electron impact broadening of spectral lines in Be-like ions: quantum calculations. J. Phys. B 2008, 41, 025702.

31. Sahal-Bréchot, S. Impact theory of the broadening and shift of specral lines due to electrons and ions in plasma(Continued). Astron. Astrophys. 1969, 2, 322-354.

32. Glenzer, S.; Uzelac, N.I.; Kunze, H.J. Stark broadening of spectral lines along the lsoelectronic sequence of Li. Phys. Rev. A 1992, 45, 8795-8802.

33. Wrubel, T.; Glenzer, S.; Buescher, S.; Kunze, H.-J.; Alexiou, S. Line profile measurements of the 2s3s-2s3p singlet and triplet transitions in Ne VII. Astron. Astrophys. 1996, 307, 1023-1028.

(c) 2014 by the authors; licensee MDPI, Basel, Switzerland. This article is an open access article distributed under the terms and conditions of the Creative Commons Attribution license (http://creativecommons.org/licenses/by/3.0/). 